\title{
Adolescent Haematocolpos: Seek and You Shall Find
}

\author{
Volha Pankevich ${ }^{1,2}$ \\ ${ }^{1}$ Emergency Department, Sunshine Coast University Hospital, Birtinya Queensland, Australia \\ ${ }^{2}$ Emergency Department, Albury Wodonga Health, Albury Campus, Albury, New South Wales, Australia
}

\section{Email address:}

Dr.Volha.Pankevich@gmail.com

\section{To cite this article:}

Volha Pankevich. Adolescent Haematocolpos: Seek and You Shall Find. Clinical Medicine Research. Vol. 10, No. 4, 2021 , pp. $112-115$. doi: $10.11648 /$ j.cmr.20211004.11

Received: June 10, 2021; Accepted: June 28, 2021; Published: July 2, 2021

\begin{abstract}
An adolescent girl presented to the emergency department with a history of abdominal pain, dribbling, and inability to pass urine for the last 24 hours. The initial observations and examination of respiratory, cardiovascular, ears, nose, and throat (ENT), central nervous system (CNS) and musculoskeletal systems were normal. No onset of menses reported by the patient. She was found to have a palpable distended bladder on abdominal examination. A catheter was inserted by the nurse, who reported no abnormalities. The physician did not perform a FAST scan nor conduct a genital examination. The patient was referred to the paediatrics department and the following day an ultrasound examination led to a diagnosis of haematometrocolpos. The patient was the referred to the obstetric and gynaecological department. Corrective surgery was conducted the following day and she was discharged on the fourth day. While the diagnosis and treatment were correct, had a FAST scan and/or genital examination been part of the initial work-up, diagnosis would have been made in the emergency department and an appropriate referral made directly to obstetric and gynaecological team. Rapid diagnosis and treatment would have benefitted the patient, reduced the risks of complications, and cut the length of stay in the hospital by as much as two days.
\end{abstract}

Keywords: Haematocolpos, Urinary Retention, FAST, Ultrasound, PoCUS

\section{Introduction}

Diagnostic delays are expensive, enhance the risks of complications, protract the patient's discomfort or pain, and contribute to bed block in the emergency department. [1-3] In complex cases, delays are often inevitable, the timing of imaging and laboratory work are not under the control of the emergency department. [4] Unnecessary delays can arise in other cases due to inadequate skills, hesitancy to take complete histories or conduct appropriate physical examinations or poor communications among staff. [5-7] This case of adolescent haematocolpos, discussed herein, illustrates how these considerations can result in very substantial delays in accurately diagnosing and treating a patient.

Paediatric presentations of acute urinary retention to the emergency department, while certainly not commonplace, are encountered with some frequency. The differentials include trauma, female genital mutilation (FGM), infection, functional disorders, neurogenic dysfunction such as acute disseminated encephalomyelitis, Guillian Barre syndrome, transverse myelitis, cauda equina, as well as tumors and congenital renal tract lesions. [8-10].

\section{Presentation}

In the early afternoon, a previously well adolescent girl presented to the emergency department with a history of abdominal pain, dribbling, and inability to pass urine for the last 24 hours. She had no history of infective symptoms, dysuria, rashes or trauma. The standard questioning using the Bristol stool chart diagrams showed no history of constipation. She reported that her menstrual periods had not yet commenced. The patient denied any vaginal discharge and there was no history of sexual activity.

\section{Investigations}

The initial observations and examination of respiratory, cardiovascular, ENT, CNS and musculoskeletal systems were normal. She was found to have a palpable distended bladder 
on abdominal examination. The nursing staff performed a bladder scan with a Verathon Bladderscan ${ }^{\circledR}$ which revealed more than one litre of retained urine. To relieve the obstruction, the physician requested that an indwelling urinary catheter (IDC) be inserted by the female nursing staff. After the insertion, no abnormalities were reported to the attending emergency department physician.

\section{Referral}

The patient was referred to the paediatric inpatient team in the late afternoon for evaluation and admission. The admitting paediatric physician reviewed the child's records an examined the patient later in the evening. He agreed to admit her to the inpatient paediatric ward and the patient was transferred shortly thereafter. During the morning rounds, on repeat abdominal examination, the patient was found to have a separate palpable mass in the surpapublic area and right iliac fossa after complete drainage of the bladder. No examination of the genitalia was undertaken but based on the findings from the abdominal examination, a formal ultrasound examination (USS) was requested and performed late in the afternoon.

\section{Diagnosis}

The findings of the USS were reported shortly thereafter and showed debris and haemorrhage within the distended vagina with minor extension into the endometrial cavity in keeping with haematometrocolpos. The uterus had normal appearance. The bladder was partially decompressed with a Foley ${ }^{\circledR}$ catheter in situ. Bladder volume during the examination was 253 millilitres. There was minor bilateral asymmetrical pelvicalyceal prominence, more prominent on the left side.

On the USS images of the lower abdomen (Figure 1), it can be seen that the distended vagina was filled with clotted blood, with the partially decompressed bladder above it. It can also be observed, in the second set of images that the uterine cavity is also distended and filled with fluid and large haematocolpos with clotted blood. (Figure 2) In light of these findings, the obstetric and gynaecological team was asked to review the patient that evening. After reviewing the patient's records and USS images, it was clear that surgical intervention was necessary. They discussed the situation and proposed management with the patient and her family. The decision was taken to schedule the surgery the following morning.

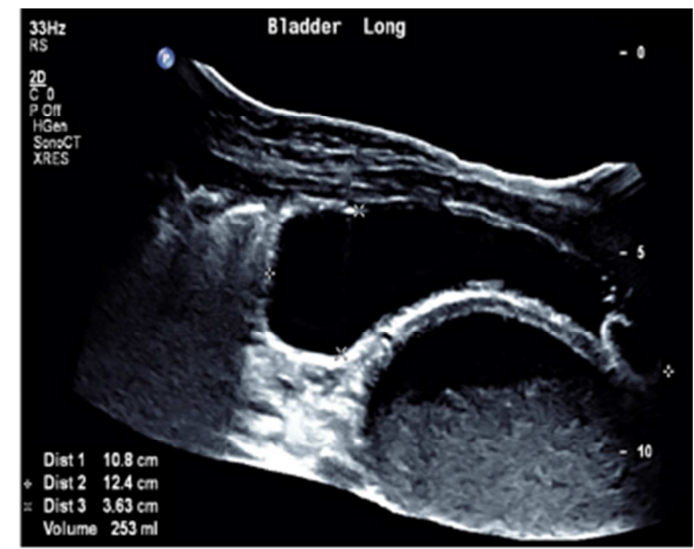

Figure 1. Transverse view of the partially emptied bladder with inflated Foley ${ }^{\circledR}$ balloon in situ and distended vagina filled with clotted blood (left) and lonitudinal view of parially filled bladder and large haematocolpos (right).
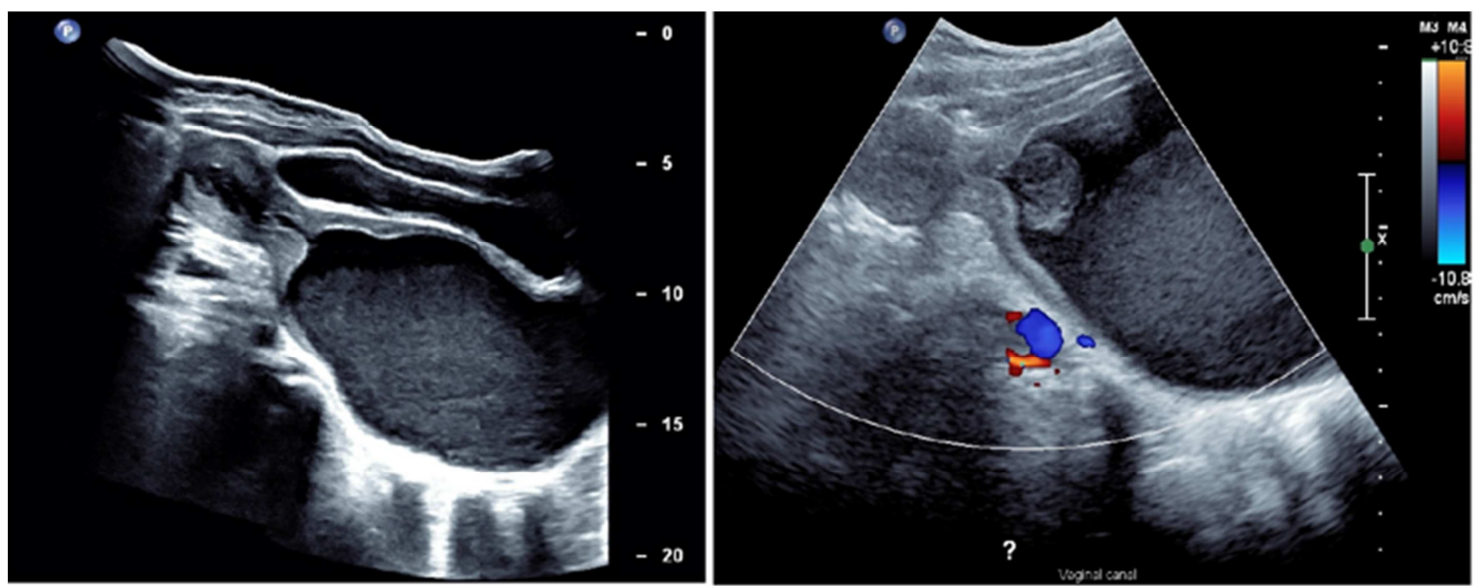

Figure 2. Lonitudinal view of the partially decompressed bladder,uterine cavity filled with fluid and large haematocolpos (left) and lonitudinal view of distended uterine cavity and large haematocolpos with clotted blood (right). 


\section{Management}

On the third day of admission, the patient underwent a 4 quadrant hymenectomy with needle point daithermy. A large haematocolpos of 650 millilitres was evacuated. No complications were encountered. The patient was discharged the following morning. She was prescribed a 5-day course of Augmentin $\AA$. A follow-up USS examination was arranged six weeks after the surgery, to confirm the resolution of the hydronephrosis.

\section{Discussion}

Failure to promptly diagnose and treat haematocolpos carries with it the risk of serious complications, including pain, peritonitis, endometriosis, which may affect fertility, and urinary tract infections. [11, 12]

The patient in this case was correctly diagnosed and properly treated. Looking at the diagnostic process in this case, three ways that treatment of similar cases might be more effectively and efficiently handled may be identified. These changes could result in earlier diagnosis, more appropriate referrals, and more expeditious corrective surgery. Earlier diagnosis and surgical intervention reduce the risk of complications, accelerates the relief of the patient's physical discomfort, and reduces the length of the hospital stay and cost of care.

Point-of-care ultrasound (PoCUS) enables “... the rapid evaluation of previous and new diagnostic hypotheses and support immediate therapeutic decisions." [13] In cases such as this one, a quick bedside Focused Assessment with Sonography in Trauma (FAST) examination of the abdomen would have revealed the same images as were obtained during the formal USS a day later. The exam consists of a transverse sweep, using the bladder as a sonographic window, so as to obtain the view of the pouch of Douglas or rectovesical space for the presence of free fluid. As can be seen in Figure 1, the collection of clotted blood in the distended vagina, located just behind the deflated bladder, is readily identified.

Armed with the knowledge gleaned from the USS, the emergency department physician would be able to make a firm diagnosis of haematocolpos, which would dictate a referral to obstetrics and gynaecology rather than to paediatrics, with the questions as to diagnosis and treatment left open. With an early diagnosis, a case such as this might have gone to the theatre for corrective surgery on the same day that the patient presented.

Although the emergency department physician in this case conducted a number of appropriate investigations, he chose not to conduct a physical examination of the patient's genitalia. This examination should be a routine part of the work-up of a patient presenting with urinary retention. It may lead to discovery of rashes, indications of infection, including STDs, congenital deformities, FGM, trauma as well as haematocolpos. In this instant case, even a cursory exam would have revealed that the problem was an imperforate hymen which would present as a bulging, protruding, bluish membrane that allows positive transillumination at the introitus.[14] This observation would also have resulted in a more appropriate referral and early treatment.

It is recognized that physicians, particularly males, are hesitant to conduct genital examinations of adolescent female patients. The examination can be uncomfortable for both the patient and the physician, though this discomfort can be minimized by following established protocols. The exam is an essential diagnostic procedure and failure to examine may result in misdiagnosis and inappropriate or delayed treatment. [15]

The nursing staff are an important part of the treatment team. In presentations of urinary retention, the insertion of an IDC is routine, and this is normally done by a nurse, as was in this case. Observation of the genitals is incidental to the procedure. There is nothing in the medical notes as to whether the nurse in this case was untrained to notice and report overt genital abnormalities or whether she assumed that the physician was already aware of the problem and felt there was no need to report what was already known. Effective communication amongst medical staff is an essential element in the provision of quality medical care.

\section{Conclusion}

While the diagnosis and treatment were correct, had a FAST scan and/or genital examination been part of the initial work-up, diagnosis would have been made in the emergency department and an appropriate referral made directly to obstetric and gynaecological team. Rapid diagnosis and treatment would have benefitted the patient, reduced the risks of complications, and cut the length of stay in the hospital by as much as two days.

\section{Learning Points}

1) FAST scans should be a routine and integral part of the investigation of urinary retention in children.

2) Genital examinations and documentation of secondary sexual characteristics should be a routine and integral part of the investigation of urinary retention in children.

3) Nursing staff should be trained to recognize, report, and record any suspected genital abnormalities observed during the course of catheterisation.

\section{Declarations}

\section{Conflicts of Interest}

The author declares that she has no competing interests.

\section{Informed Consent}

Not applicable / no identifiers. 


\section{Author Contributions}

Dr. Volha Pankevich is the sole author.

\section{Data Availability}

Data sharing is not applicable to this article as no new data were created or analyzed in this study.

\section{References}

[1] Shekelle P, Pronovost P, Wachter R, Taylor S, Dy S, Foy R, et al. Advancing the science of patient safety. Ann Intern Med. 2011; 154: 693-96.

[2] Newman-Toker D, Wang Z, Zhu Y, Nassery N, Saber Tehrani A, Schaffer A, et al. Rate of diagnostic errors and serious misdiagnosis-related harms for major vascular events, infections, and cancers: toward a national incidence estimate using the "Big Three". Diagnosis. 2021; 8 (1): 67-84.

[3] Huang Q, Thind A, Dreyer, J, Zaric G. The impact of delays to admission from the emergency department on inpatient outcomes. BMC Emerg Med (2010); 10, 16. doi: $10.1186 / 1471-227 X-10-16$.

[4] Caesar U, Karlsson J, Hansson E. Incidence and root causes of delays in emergency orthopaedic procedures: a single-centre experience of 36,017 consecutive cases over seven years. Patient Saf Surg. 2018; 12: 2. doi: 10.1186/s13037-018-01491.

[5] Hussain F, Cooper, A, Carson-Stevens A, Donaldson L, Hibbert P, Hughes T, et al. Diagnostic error in the emergency department: learning from national patient safety incident report analysis. BMC Emerg Med 2019: 19, 77. doi: 10.1186/s12873-019-0289-3.
[6] Bari A, Khan R, Rathore A. Medical errors; causes, consequences, emotional response and resulting behavioral change. Pak J Med Sci. 2016; 32 (3): 523-28.

[7] Verghese A, Charlton B, Jerome P. Kassirer J, Ramsey M, Ioannidis J. Inadequacies of physical examination as a cause of medical errors and adverse events: a collection of vignettes. AMJ Med. 2015; 128 (12): 1322-24.

[8] Asgari S, Mansour Ghanaie, M., Simforoosh, N., Kajbafzadeh, A., Zare', A., Acute urinary retention in children. Urol J. 2005; 2 (1): 23-27.

[9] Effa E, Ojo, O., Ihesie, A., Meremikwu, M., Deinfibulation for treating urologic complications of type III female genital mutilation: A systematic review. Int J Gynecol Obstet 2017; 136: 30-33.

[10] Gatti J,Perez-Brayfield, M., Kirsh, A., Smith, E., Massad, H., Acute urinary retention in children. J Urol 2001; 2013: 918-21.

[11] Posner J, Spandorfer, P., Early detection of imperforate hymen prevents morbidity from delays in diagnosis. Pediatrics 2005; 115 (4): 1008-12.

[12] Lardenije C, a cause of abdominal pain in female adolescents. Case Reports, 2009 (may26 1): bcr0820080722bcr0820080722.

[13] Cormac C, Wald, A., Coombs, P., Kallos, L. and Blecher, G., Time to establish pillars in point-of-care ultrasound. Australasian Journal of Ultrasound in Medicine 2019; 22 (1): 12-14.

[14] Kotter H, Weingrow, D., Canders, C., Hematometrocolpos in a pubescent girl with abdominal pain. Clin Pract Cases Emerg Med 2017; 1 (3): 218-20.

[15] Horner G, Genitourinary assessment: an integral part of a complete physical examination. J Pediatr Health Car 2007; 21 (3): 162-70. 